\title{
Eyelid Localization in Iris Images Captured in Less Constrained Environment
}

\author{
Xiaomin Liu, Peihua Li, and Qi Song \\ College of Computer Science and Technology, Heilongjiang University \\ Harbin, Hei Long Jiang Province, 150080, China \\ peihualj@hotmail.com
}

\begin{abstract}
Eyelid localization plays an important role in an accurate iris recognition system. In less constrained environment where the subjects are less cooperative, the problem becomes very difficult due to interference of eyelashes, eyebrows, glasses, hair and diverse variation of eye size and position. To determine upper eyelid boundary accurately, the paper proposes an integro-differential parabolic arc operator combined with a RANSAC-like algorithm. The integro-differential operator works as a parabolic arc edge detector. During search process of the operator, the potential candidate parabolas should near at least certain percentage of edgels of upper eyelid boundary, detected by 1D edge detector. The RANSAC-like algorithm functions as a constraint that not only makes eyelid localization more accurate, but also enables it more efficient by excluding invalid candidates for further processing. Lower eyelid localization is much simpler due to very less interference involved, and a method is presented that exploits $1 \mathrm{D}$ edgels detection and an RANSAC algorithm for parabolic fitting. Experiments are made on UBIRIS.v2 where images were captured at-a-distance and on-the-move. The comparison shows that the proposed algorithm is quite effective in localizing eyelids in heterogeneous images.
\end{abstract}

Keywords: Eyelid localization, Integro-differential parabolic arc operator, RANSAC, 1D Signal.

\section{Introduction}

Iris recognition has received increasing interest [127/3] among many biometrics technologies, such as face, fingerprint and speech recognitions etc. The characteristics that distinguish iris recognition from others include enormous statistical variability of iris patterns among different persons, stability over time of iris structure, non-invasiveness to human user and high recognition rate [5]6].

Accurate iris segmentation is a prerequisite step for a successful iris recognition system. In iris segmentation, after limbic boundary and pupillary boundary are determined, it is necessary to further localize upper and lower eyelids so that it could be determined whether or not iris is occluded. The straightforward idea is either to simply exclude the top or bottom parts of iris [5] or to use straight lines to fit eyelid boundaries [1011 and invisible iris regions are 
removed if obstruction occurs. Wildes [12 proposed a two-stage method for both limbic and eyelid boundaries localization, which consists of edge detection followed by Hough transform, tuned for circular and parabolic arcs respectively. In [4], eyelash region is firstly segmented by using high frequency information based on wavelet transform. Next raw eyelid contour, modeled by a parabolic arc, is achieved with least square fitting technique, and at last the eyelid position is fined. Jang et al. 9] introduced a robust eyelid detection approach that could deal with both defocused iris images and rotated iris images. Their method used different masks for eyelid boundary points detection according to focus assessment, and adopted a Hough transform to fit a parabola.

In less constrained environment [1, iris images are captured at-a-distance and on-the-move and eyelid boundary localization becomes more difficult, because of impact of eyelashes, eyebrows, hair, glasses and diverse variation of eye size and position. Fig. 1] shows some challenging examples of iris images.

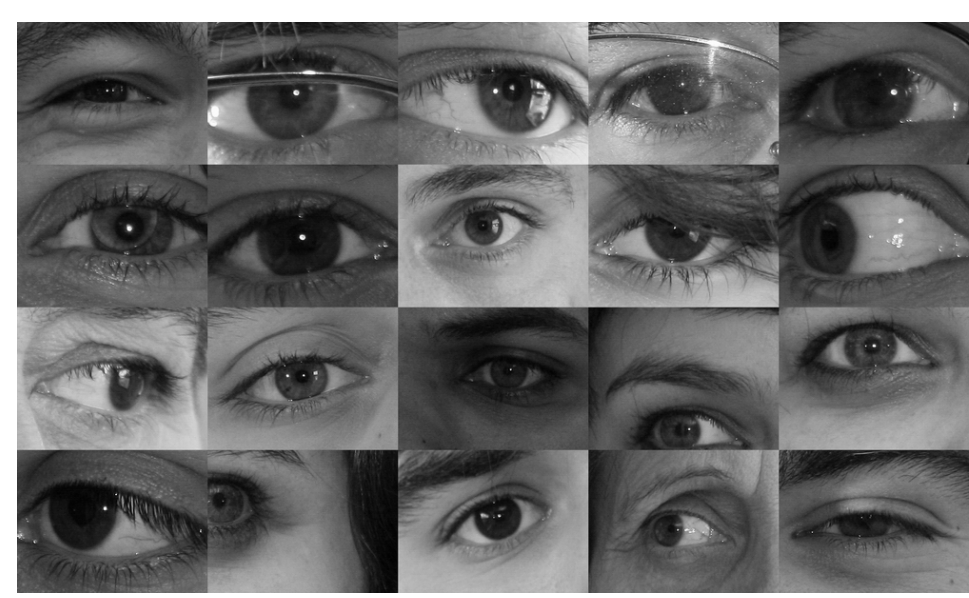

Fig. 1. Some iris images in UBIRIS.v2 database [1]

We focus on eyelid detection in these heterogeneous images. The limbic boundary is firstly localized using an improved Daugman's method [5] that is not discribed here due to page limit. Then we use an integro-differential parabolic arc operator for upper eyelid detection. During searching process of the ideal parabola, we introduce a RANSAC-like [8] algorithm to constrain the candidate parabolas. The constraint is that a valid potential parabola should near at least certain percentage of eyelid edgels detected by $1 \mathrm{D}$ edge detector, considering the fact that edgels may be noisy and not accurate. The resulting algorithm becomes more accurate and in the meantime more efficient thanks to exclusion of invalid candidates. In contrast to localization of upper eyelid, that of lower eyelid is much simpler and a straightforward method is briefly described. 


\section{Our Approach}

In this section, we first consider problem of upper eyelid detection in sections 2.1 to 2.3 and then describe an approach for lower eyelid detection in section 2.4.

\subsection{Integro-Differential Operator for Upper Eyelid Detection}

The upper eyelid is modeled by a parabola of the form

$$
\Gamma(a, b, c): \quad y=a(x-b)^{2}+c
$$

Given the outer circle, characterized by the center $\left(x_{c}, y_{c}\right)$ and the radius $R$, of the iris localized by a method that is not described here due to page limit, we can determine the ranges of three parameters $a, b$ and $c$. The parabola should open to the bottom and its curvature should be less than that of the iris, thus $a$ should be in the interval $[0,1 /(2 R)]$. According to geometric relationship of eyelid and iris, we can further constrain the domains of the parabola vertex $(b, c)$ : $b \in\left[x_{c}-R / 2, x_{c}+R / 2\right], c \in\left[y_{c}-3 R / 2, y_{c}+2 R / 3\right]$.

Motivated by Daugman's paper [5], an integro-differential operator for parabolic location is proposed as follows

$$
\underset{a, b, c}{\operatorname{argmax}} f(a, b, c)=G_{\sigma}(c) * \frac{\partial}{\partial c} \int_{\Gamma(a, b, c)} \frac{I(x, y)}{L(a, b, c)} d s
$$

where $I(x, y)$ is the iris image, the symbol $*$ stands for convolution operation, $G_{\sigma}(c)$ is a Gaussian with standard deviation $\sigma$, and $L(a, b, c)$ denotes the arclength of the parabola $\Gamma(a, b, c)$. The integro-differential operator can be interpreted as a parabolic arc edge detector, which searches the parameter spaces of the parabola along which the smoothed integral derivative is maximum. Notice that when occlusion occurs the eyelid boundary above the iris may be weak and difficult to distinguish, thus the segment of the parabolic arc within the region as shown in Fig. 22 is excluded when calculating curve integral with Eq. (2).

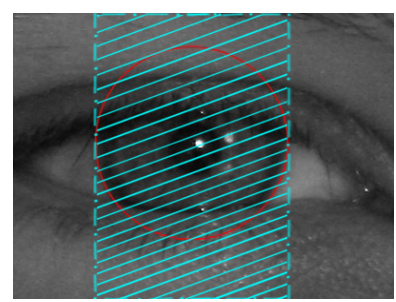

Fig. 2. The segment of a parabolic arc that falls within the shaded region is removed when evaluating curve integral with Eq. (2) 


\subsection{D Edgels Detection of Upper Eyelid Boundary}

Given the iris position $\left(x_{c}, y_{c}\right)$ and radius $R$, we can detect eyelid boundary edgels along line segments

$$
\begin{aligned}
& x=d \\
& y_{c}-2 R \leq y \leq y_{c}+R / 2
\end{aligned}
$$

where $d$ is constant varying in intervals $\left[x_{c}-3 R, x_{c}-R / 2\right]$ or $\left[x_{c}+R / 2, x_{c}+3 R\right]$. Firstly 1D signal is extracted according to Eq. (3), which is then smoothed with Gaussian, finally the gradient is computed and the point of maximum gradient that is larger than a prescribed threshold $\varepsilon_{e}\left(\varepsilon_{e}=3.0\right.$ in our case) is regarded as an edgel of the eyelid boundary. Fig. 3 shows an example, where Fig. 3(a) gives search region of 1D signal. Figs. 3(b), 3(c) and 3(d) provide respectively a line segment along which 1D signal is extracted, filtered 1D signal and the gradient of the filtered 1D signal. The Edgels, $\mathbf{p}_{i}, i=1, \cdots, N_{e}$, and the final parabolic arc denoting the upper eyelid boundary are given in Figs. 3(e) and 3(f) respectively. Note that only a portion of detected edgels is accurate, please refer to Fig. 3(e)

\subsection{The Proposed Algorithm for Upper Eyelid Boundary Localization}

The proposed algorithm is illustrated in Algorithm 1, The search ranges of parameters $a, b, c$ are discretized by $s t e p \_a, s t e p \_b, s t e p \_c$ respectively. For each three-tuple $(a, b, c)$ in the search spaces, a RANSAC-like algorithm is used to determine whether or not the three-tuple is accepted as a potential candidate or just rejected. Given $(a, b, c)$, the number $N_{p}$ of detected edgels $\mathbf{p}_{i}, i=1, \cdots, N_{e}$, is counted for which the distance of the edgel to the parabola is below a threshold $\epsilon_{d}$. If the percentage of $N_{p}$ over $N_{e}$ is above another threshold $\epsilon_{N}$, the parabola characterized by the three-tuple is a valid candidate for which $f(a, b, c)$ is computed according to Eq. (2). Finally we can get the parabola characterized by the three-tuple $\left(a^{*}, b^{*}, c^{*}\right)$ with maximum $f$. In our experiments, $\varepsilon_{d}=15.0$ pixels, $\epsilon_{N}=0.5$.

The underlying idea is that at least a portion $\epsilon_{N}$ of edgels, detected as described in section 2.2, is accurate that functions as constraint through a RANSAC-like algorithm. The accurate here means that the distance between a pixel and the parabolic arc is within a tolerance $\varepsilon_{d}$ pixels. The constraint imposed in this way makes eyelid boundary localization more accurate. An additional advantage is that the proposed algorithm becomes more efficient, because the parabolas that do not satisfy the constraint condition are excluded from further processing.

\subsection{Locating the Lower Eyelid}

Compared to upper eyelid detection, it is much simpler to detect lower eyelid due to less interference, please refer to Fig. 1. At the beginning, we perform $1 \mathrm{D}$ 


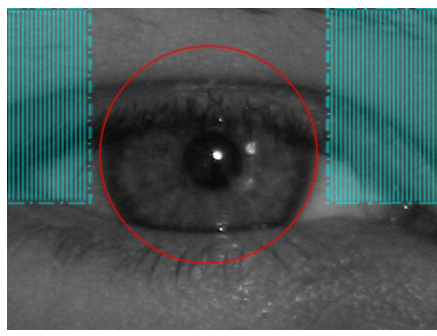

(a) Search region of $1 \mathrm{D}$ signal

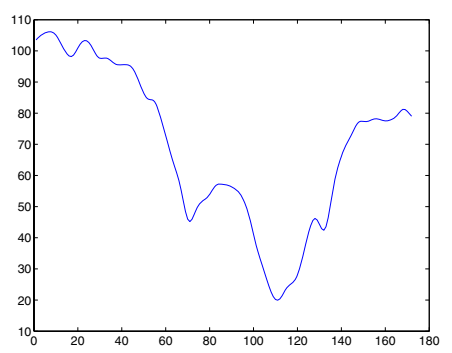

(c) 1D signal filtered with Gaussian

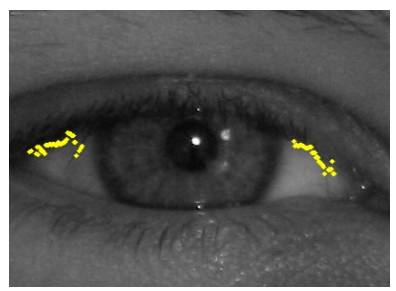

(e) Edgels obtained with 1D search

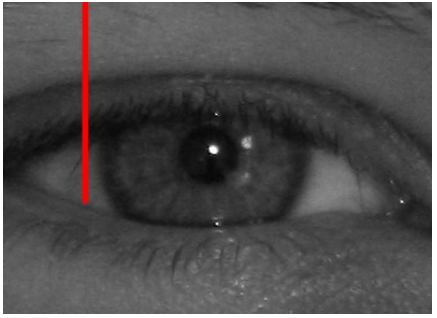

(b) Line segment to extract 1D signal

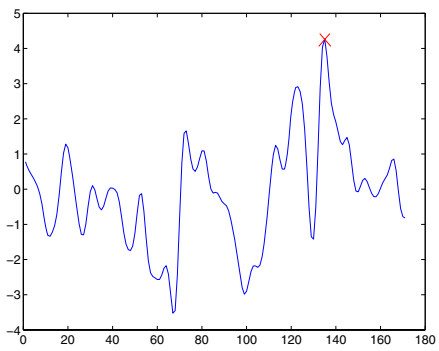

(d) Gradient of 1D signal

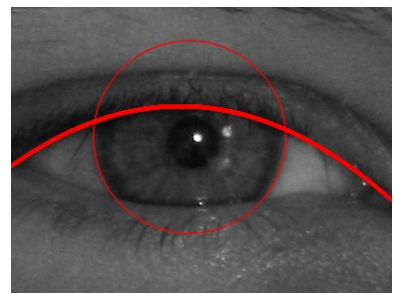

(f) Localization result

Fig. 3. Upper eyelid localization

search to detect edgels of eyelid boundary. The 1D search is along line segments of the form

$$
\begin{aligned}
& x=d \\
& y_{c} \leq y \leq y_{c}+3 R / 2
\end{aligned}
$$

where $d$ is constant varying in the interval $\left[x_{c}-R / 2, x_{c}+R / 2\right]$. Please note the difference of Eq. (4) from Eq. (3). Because the edgels detected in this way may be noisy, RANSAC algorithm [8] is used to fit parabolic arc. Fig. 4 shows two examples of lower eyelid boundary detection, where the dashed rectangle indicates searching area of 1D signal, and the yellow dots represent edgels detected. Please note that when iris is not obstructed by lower eyelid, we actually detect lower boundary of iris, as demonstrated in Fig. 4(c). In both cases, we can determine whether or not occlusion happens and then remove occlusion regions, if any, as 


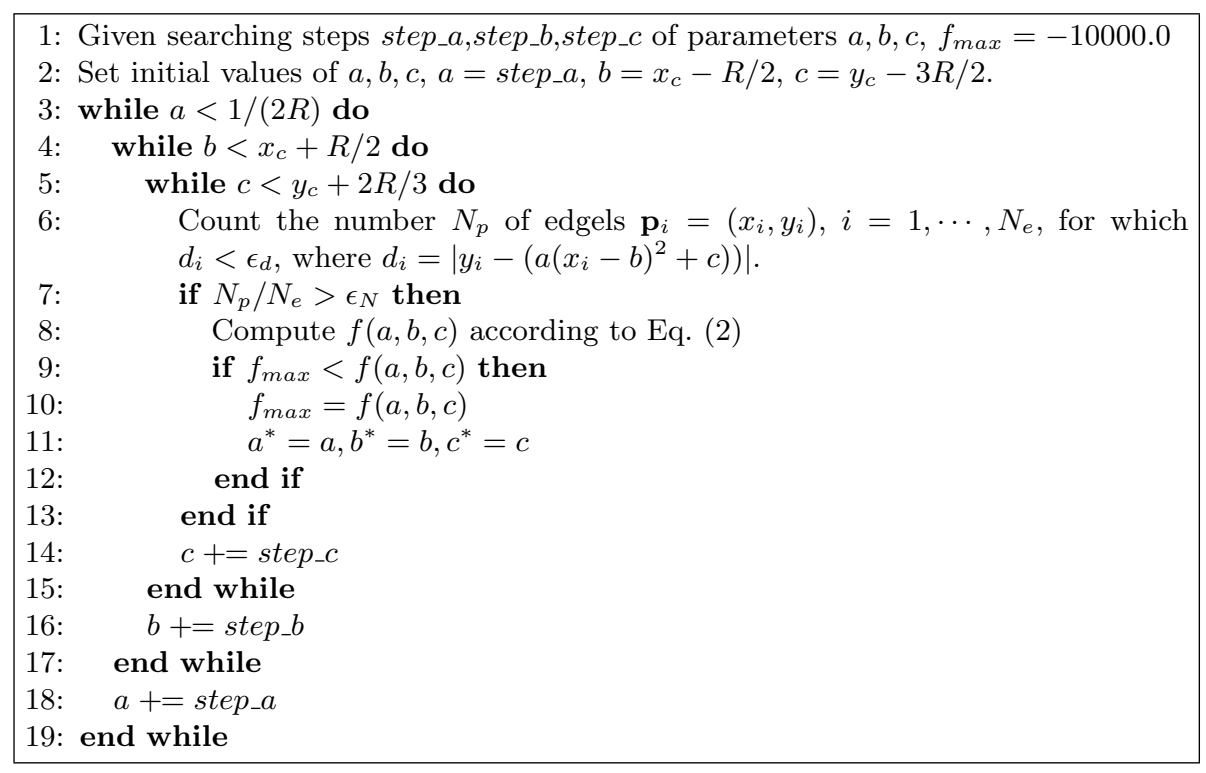

Algorithm 1. The pseudocode of the proposed algorithm to locate upper eyelid

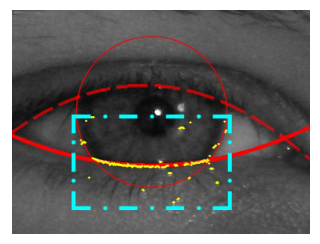

(a)

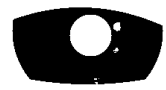

(b)

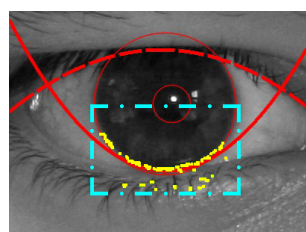

(c)

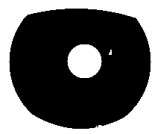

(d)

Fig. 4. Eyelid detection and occlusion removal. Explanation please refer to text.

shown in Figs. 4(b) and 4(d). Note that in the latter case (as shown in Figs. 4(c) and $4(\mathrm{~d})$ there is no occlusion indeed that can be determined correctly.

\section{Experiments}

The algorithm is implemented with $\mathrm{C}++$ on a $\mathrm{PC}$ with $3.0 \mathrm{GHz}$ Intel Pentium(R) $4 \mathrm{CPU}$ and 2G Memory, and we do not make any code optimization. We primarily evaluate the performance of the proposed upper eyelid localization algorithm, comparing it with several other methods as described in Table 1. Because lower eyelid is much simpler to localize, in all algorithms the same method as described in section 2.4 is used for lower eyelid detection. 
Table 1. Description of algorithms to be compared with the proposed one

\begin{tabular}{ll}
\hline Algorithm description \\
\hline Algorithm A & $\begin{array}{l}\text { Algorithm A depends only on brute force search with integro- } \\
\text { differential operator as described in section 2.1 }\end{array}$ \\
\hline Algorithm B & $\begin{array}{l}\text { Algorithm B is dependent merely on edgels obtained with 1D } \\
\text { methods as explained in section [2.2, and uses least square } \\
\text { fitting to get the parabola }\end{array}$ \\
\hline Algorithm C & $\begin{array}{l}\text { Algorithm C counts only on edgels obtained with 1D methods } \\
\text { as explained in section 2.2 as well, but, unlike algorithm B, } \\
\text { uses RANSAC-like algorithm to fit the parabola }\end{array}$ \\
\hline Algorithm D & $\begin{array}{l}\text { Algorithm D relies on Canny edge detection followed by } \\
\text { Hough transform for parabolic detection }\end{array}$ \\
\hline
\end{tabular}

\subsection{Database and Ground Truth}

We use UBIRIS.v2 iris database [1] in which images were captured at-a-distance and on-the-move. Due to the less constrained environment, the iris images are very heterogeneous and difficult for segmentation due to severe specular highlight, hair and glasses occlusion, considerable variation of eye size and position, eyelashes and eyebrows.

There are a total of 500 iris images of size 400x300 in UBIRIS.v2, in which 29 images are excluded that contains either closed-eyes or rotated-eyes, and the remaining 471 images are used for experiment. The thresholds involved in our algorithm are tuned on 100 images randomly selected in the set of 471 images. For the $i$ th one, $i=1,2, \cdots, 471$, we hand-labeled the upper-eyelid boundary points and least square fitting is taken to estimate a parabolic arc that is used as the ground truth $y_{t, i}$, and the interval $\left[x_{1}, x_{2}\right]$ of the ground truth $y_{t, i}$ is determined simultaneously by the experimenter. The $L^{2}$-norm [2] in the following form is adopted to measure the error between the ground truth and an observation

$$
E_{i}=\left\|y_{t, i}-y_{o, i}\right\| \triangleq\left(\frac{1}{x_{2}-x_{1}} \int_{x_{1}}^{x_{2}}\left(y_{t, i}-y_{o, i}\right)^{2} d x\right)^{\frac{1}{2}}
$$

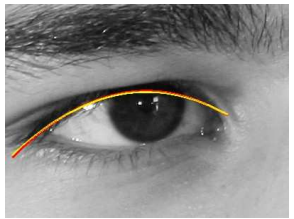

(a) 1.0336

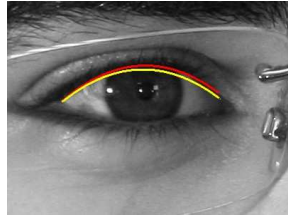

(b) 5.1518

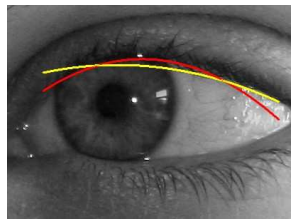

(c) 10.7277

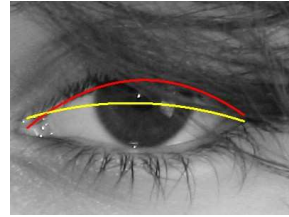

(d) 23.6786

Fig. 5. Four examples of upper eyelid localization error between the ground truth (the red parabolic arc) and the observation (the yellow one), measured by Eq. (5). From left to right, the errors are 1.0336, 5.1518,10.7277 and 23.6786 pixels respectively. 
Table 2. Comparison of upper eyelid localization

\begin{tabular}{lcc}
\hline Algorithm & Average error (pixels) & Average time (s) \\
\hline Proposed algorithm & 12.09 & 0.424 \\
Algorithm A & 15.27 & 1.848 \\
Algorithm B & 34.39 & 0.037 \\
Algorithm C & 99.98 & 0.037 \\
Algorithm D & 24.85 & 0.746 \\
\hline
\end{tabular}

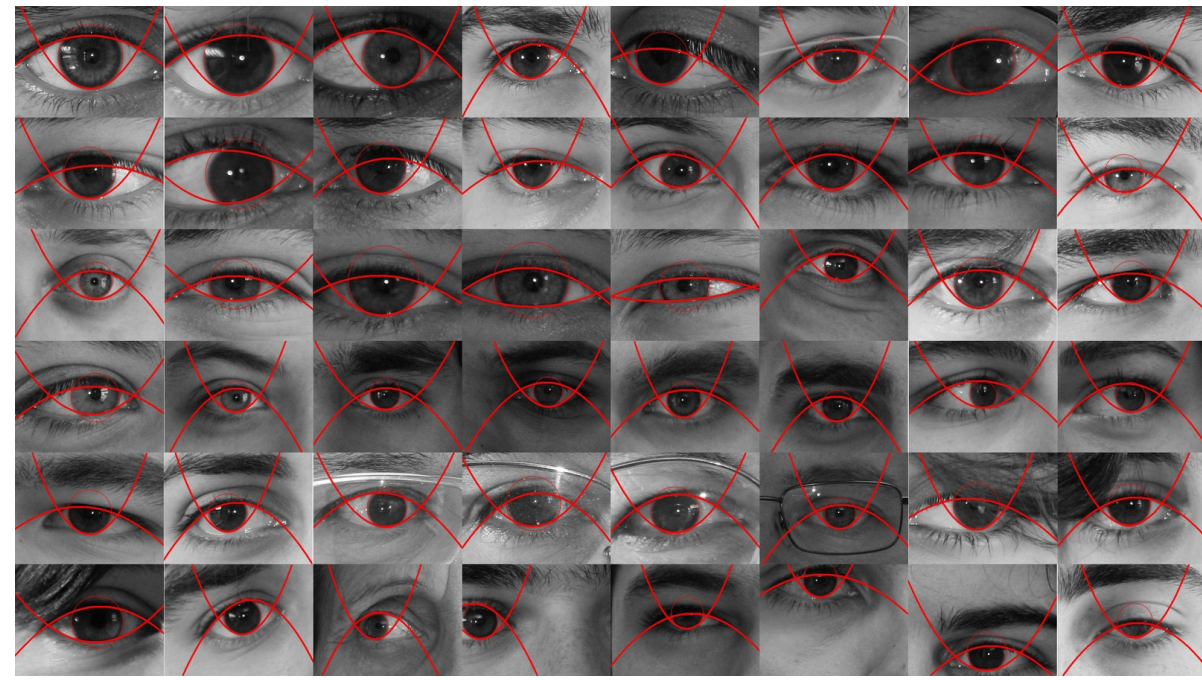

(a) Examples of good localization

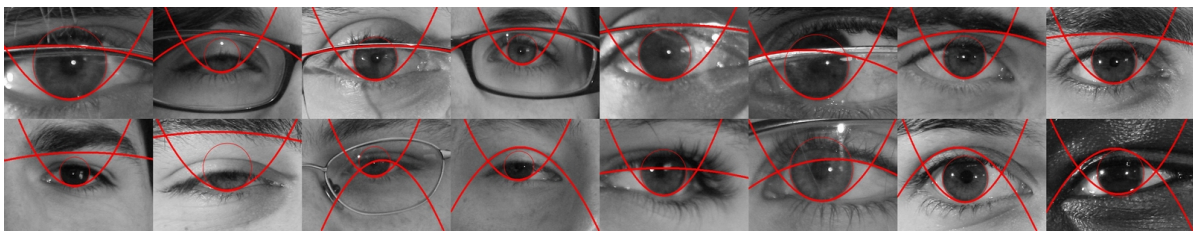

(b) Examples of bad localization

Fig. 6. Examples of eyelid localization with the proposed algorithm

where $y_{o, i}$ denotes the parabola detected in the $i$ th iris image with the proposed algorithm. Then the average error can be computed

$$
\bar{E}=\frac{1}{N} \sum_{i=1}^{N}\left\|y_{t, i}-y_{o, i}\right\|
$$


where $N$ is the total number of images. Fig. 5 presents four examples of upper eyelid localization error, where the red and the yellow parabolic arcs denote the ground truth and the observation respectively. From left to right, the errors measured by Eq. (5) are 1.0336, 5.1518,10.7277 and 23.6786 pixels respectively.

\subsection{Comparison Result}

Comparison of the proposed algorithm with other ones for upper eyelid localization is presented in Table 2, Above all, we notice that the proposed algorithm is significantly better than Algorithm D-Algorithm of Canny edge detection plus Hough transform, in both accuracy and computing time. Compared to the proposed algorithm, Algorithm A, which depends only on exhaustive search with integro-differential operator, deteriorates in localization accuracy but consumes much more time. Algorithm B that is dependent solely on least square fitting of eyelid edgels demonstrates bad results though it is quite efficient. Finally, Algorithm C, relying only on RANSAC-like fitting of eyelid edgels, exhibits surprisingly large errors. The reason is that, according to our analysis, the number of edges is small due to threshold $\varepsilon_{t}$ we used, and the RANSAC-like algorithm does not work in most cases.

Some typical examples of eyelid localization with the proposed algorithm is shown in Fig. 6, where Fig. 6(a) provides examples of good localization while Fig. 6(b) gives those of bad localization. Boundaries of glasses or eyebrows, inaccurate localization of outer iris, low contrast between sclera region and eyelid can account for most of failure localization.

\section{Conclusions}

The paper addresses eyelid detection problem in heterogeneous images captured at-a-distance and on-the-move. In this type of less constrained environment, eyelid boundary localization becomes more difficult, due to interference of eyelashes, eyebrows, hair, glasses and more diverse variation of eye size and position. The proposed method for upper eyelid detection consists in an integro-differential parabolic arc operator combined with a RANSAC-like algorithm that imposes constraint of edgels on the operator. Lower eyelid detection is much simpler due to few interference involved, and a straightforward approach is developed. Current work can not deal with cases of closed-eye and rotated-eye that is left for our future research. We would also like to test the performance of our algorithm on other well-known iris databases.

\section{Acknowledgements}

The work was supported by the National Natural Science Foundation of China under Grant 60673110 and Natural Science Foundation of Heilongjiang Province (F200512), supported in part by Program for New Century Excellent Talents of 
Heilongjiang Province (1153-NCET-002), Sci. \& Tech. Research Project of Educational Bureau of Heilongjiang Province (1151G033), the Scientific Research Foundation for the Returned Overseas Chinese Scholars, State Education Ministry and Ministry of Personnel of China, Sci. and Tech. Innovation Research Project (2006RFLXG030) of Harbin Sci. \& Tech. Bureau.

\section{References}

1. UBIRIS.v2 database (2008), http://iris.di.ubi.pt/ubiris2.html

2. Blake, A., Isard, M.: Active Contours. Springer, London (1998)

3. Bowyer, K.W., Hollingsworth, K., Flynn, P.J.: Image understanding for iris biometrics: a survey. Comp. Vis. Image Understanding 110(2), 281-307 (2008)

4. Cui, J., Wang, Y., Tan, T., Sun, Z.: A fast and robust iris localization method based on texture segmentation. In: Proc. of SPIE, pp. 401-408 (2004)

5. Daugman, J.G.: High confidence visual recognition of persons by a test of statistical independence. IEEE Trans. Pattern Anal. Mach. Intell. 15(11), 1148-1160 (1993)

6. Daugman, J.G.: The importance of being random: Statistical principles of iris recognition. Pattern Recognition 36(2), 279-291 (2003)

7. Daugman, J.G.: New methods in iris recognition. IEEE Trans. Systems, Man, Cybernetics B 37(5), 1167-1175 (2007)

8. Fischler, M.A., Bolles, R.C.: Random sample consensus: A paradigm for model fitting with applications to image analysis and automated cartography. Comm. of the ACM 24, 381-395 (1981)

9. Jang, Y.K., Kang, B.J., Park, K.R.: A study on eyelid localization considering image focus for iris recognition. Pattern Recognition Letters 29(1), 1698-1704 (2008)

10. Liu, X., Bowyer, K.W., Flynn, P.J.: Experiments with an improved iris segmentation algorithm. In: IEEE Workshop on Automatic Identification Advanced Technologies (AutoID), pp. 118-123 (2005)

11. Masek, L.: Recognition of human iris patterns for biometric identification. Bachelors Thesis (2003)

12. Wildes, R.P.: Iris recognition:an emerging biometric technology. Proc. IEEE 85(9), 1348-1363 (1997) 